
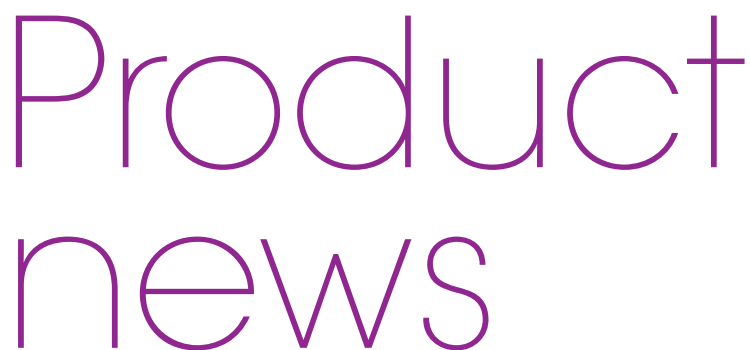

\section{TEPE GIVES SUGAR A POSITIVE SPIN}

[TePe]

At BDIA Showcase TePe unveiled its new bio-based plastic toothbrush which has been made using sugar cane and castor oil. Sustainably produced in Sweden using $100 \%$ green energy, the toothbrush is the first in the $\mathrm{TePe} \mathrm{GOOD}^{\circledR}$ range.

The use of renewable raw materials, such as sugar cane and castor oil, enables TePe to recirculate up to $95 \%$ of the $\mathrm{CO}_{2}$ emissions during the product's life cycle. As with all TePe toothbrushes, the product is manufactured at TePe's Malmö factory, which has the largest solar power plant in the city.

Elaine Tilling, Head of Clinical

Education at TePe UK, said

'The TePe GOOD' product range marks a milestone in our continued journey towards a more sustainable future. The introduction of our bio-plastic toothbrush offers an environmentally-sensitive oral care solution that does not compromise on quality. This combined with our commitment to manufacture with $100 \%$ green energy marks another important step for us as we explore opportunities to become a more sustainable business.'

The TePe GOOD ${ }^{\circ}$ toothbrush goes on sale to dental practices and surgeries in November 2018 and has a suggested retail or more detail please contact your local dental wholesaler. price $£ 2.75$. For orders

\section{MASTER THE ART OF DECONTAMINATION}

Creating a compliant decontamination process is an art form. Just as an artist has to decide how to apply light and shadow, and colour and texture, there are many facets that need to be considered to achieve complete compliance.

If you're struggling to master perfection, Little Sister can help. The Little Sister range of autoclaves, handpiece cleaner and oiler, reverse osmosis water system and essential testing products offer an effective, reliable and affordable solution for high-quality decontamination and peace of mind.

Plus, you get so much more included in the picture when you choose Little Sister, such as Lifetime Breakdown Protection, Care\&Cover Protection, free installation and commissioning, and free validation and PSSR testing. So what are you waiting for? Master the art of decontamination with Little Sister. For more information on decontamination equipment and products from EschmannDirect, please visit www.eschmann.co.uk or call Martin Wanendeya, Adrian Binney and Eimear O'Connell.

They cover a range of relevant topics including peri-implantitis, digital smile design, implants for periodontic patients, and digital planning for in-house milling of final restorations. Offering more than just clinical insight, there is even a webinar exploring the benefits of a hygiene-led team approach to implant and periodontal maintenance. ADI webinars are just one advantage available to members - find out more today.

For more information or to join the ADI, visit www.adi.org.uk/membership 01903753322

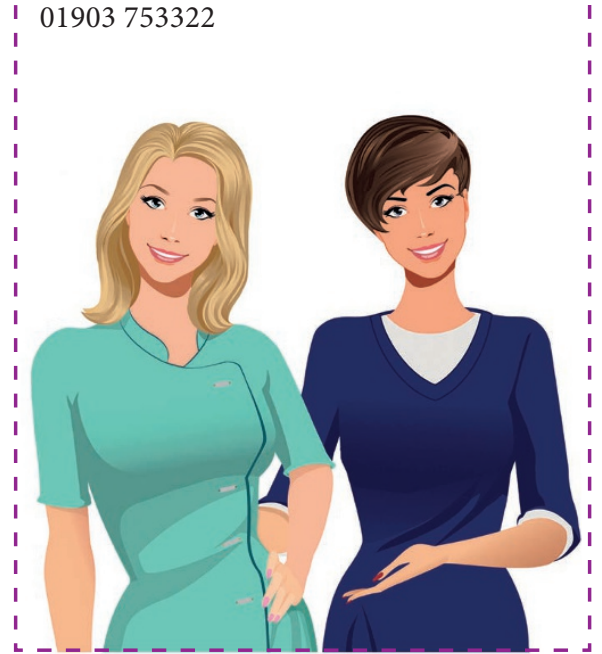

